Seung Joon Park

Sachin Shanbhag

Ronald G. Larson

\section{A hierarchical algorithm for predicting the linear viscoelastic properties of polymer melts with long-chain branching}

Received: 8 April 2004

Accepted: 11 August 2004

Published online: 5 October 2004

(C) Springer-Verlag 2004

\section{S. J. Park $\cdot$ S. Shanbhag}

R. G. Larson $(\square)$

Department of Chemical Engineering,

University of Michigan, Ann Arbor,

MI 48109, USA

e-mail: rlarson@umich.edu

Tel.: + 1-734-9360772

Fax: + 1-734-7630459

Present address: S. J. Park

Corporate Research \& Development,

LG Chem, Ltd./Research Park, 104-1,

Moonji-dong, Yuseong-gu,

Daejeon, 305-380, Korea

\begin{abstract}
The "hierarchical model" proposed earlier [Larson in Macromolecules $34: 4556-4571,2001]$ is herein modified by inclusion of early time fluctuations and other refinements drawn from the theories of Milner and McLeish for more quantitative prediction. The hierarchical model predictions are then compared with experimental linear viscoelastic data of well-defined long chain branched 1,4-polybutadienes and 1,4-polyisoprenes using a single set of parameter values for each polymer, which are obtained from experimental data for monodisperse linear and star polymers. For a wide range of monodisperse branched polymer melts, the predictions of the hierarchical model for monodisperse
\end{abstract}

melts are very similar to those of the Milner-McLeish theories, and agree well with experimental data for many, but not all, of the branched polymer samples. Since the modified hierarchical model accounts for arbitrary polydispersity in molecular weight and branching distributions, which is not accounted for in the Milner-McLeish theories, the hierarchical algorithm is a promising one for predicting the relaxation of general mixtures of branched polymers.

Keywords Long-chain branched polymers $\cdot$ Dynamic dilution theory $\cdot$ Tube model $\cdot$ Linear viscoelastic properties $\cdot$ Polydispersity

\section{Introduction}

It has long been known that rheological properties, and therefore processing properties, of polymers are highly affected by the degree of long-chain branches (LCB) [8, $9,34]$. It is therefore very important to detect and to characterize LCB. While ${ }^{13}$ C-NMR and solution techniques using light scattering combined with gel permeation chromatography (GPC) have been widely used for characterizing LCB, they have difficulty in detecting low levels of LCB, which are nevertheless able to affect drastically the rheological properties $[12,25]$. Thus, rheological methods have been developed for detecting LCB because of its sensitivity to the presence of small levels of LCB [1, 11, 29, 33].
Because the long-chain branching structure of commercial polymers, i.e., the density of branch points, branch length, and the locations of the branches along the polymer backbone or along the other branches, is complicated, it is difficult to predict their effect on the rheological properties of polymers. Recent molecular theories for long-chain-branched polymers are based on the idea of dynamic tube dilation (DTD), which models the constraint release of the entangled branched polymer melts as an effective widening of the tube. These theories are able to predict the relaxation behavior of well-defined branched polymers such as monodisperse star, $\mathrm{H}$, pom-pom, and comb polymers $[2,16-18,20]$. Despite this success, the DTD theory has been criticized for its apparent lack of controlled renormaliza- 
tion at different timescales of the effective diffusion constant of the retracting star arm [15]. In addition, the DTD theory fails to predict some experimental findings such as the relationship between the self-diffusion constant and the viscoelastic terminal time of star polymer melts and the peak of the dissipative part of the dielectric relaxation in the terminal region $[6,32]$. However, the DTD theory predicts well the stress relaxation of various ideal branched polymers and this motivates our interest in extending it to commercial polymers with complex long-chain branching structures.

To describe commercial branched polymer melts, theories of DTD developed for monodisperse polymers must be generalized so that mixed systems can be considered that are not only polydisperse in molecular mass but also polydisperse in branch length and branch placement. In addition, to be most useful, the parameters used for prediction of simple branched polymers such as monodisperse star polymers should not be readjusted when more complex branched structures are modeled.

To this end, Larson [13] developed an algorithm that generalizes the Milner-McLeish theories [2, 17-20] to predict the relaxation of general mixtures of branched polymers; it is called the "hierarchical model" because it is based on the hierarchical character of relaxation in branched polymers. Predictions of the original hierarchical model showed reasonably good agreement with experimental data for various nearly monodisperse branched structures, and the model could predict nearly quantitatively the relaxation behavior of a mixture of branched and linear polymers. However, while the original hierarchical model is based on the Milner-McLeish theory, its predictions for specific systems such as monodisperse linear and starlinear blends do not match perfectly with the predictions of the Miner-McLeish theory due to neglect of the early-time fluctuations of arm relaxation and the crude prefactor in the equation for late-time retraction in the hierarchical model [13]. In addition, besides the equilibration time $\tau_{\mathrm{e}}$, which is a fitting parameter in the Milner-McLeish theory, the original hierarchical model needs an additional fitting parameter $\tau_{0}$, which is the time constant in the prefactor of the expression for late-time fluctuations.

In this work we modify the hierarchical model to include the early-time fluctuations and to eliminate the need for the additional parameter $\tau_{0}$. We then compare its predictions with experimental data for nearly monodisperse 1,4-polybutadienes and 1,4-polyisoprenes. For branched polymers, polydispersity affects the relaxation behavior more strongly than for linear polymers because the arm relaxation time is exponentially dependent on the arm molecular weight. Thus, the small levels of polydispersity normally obtained even for anionically polymerized polymers can change the relaxation behavior drastically. In this work we also show how polydispersity in overall molecular weight and in branch molecular weight affects the rheological properties of branched polymers. We obtain a single set of parameter values for each polymer by fitting data for monodisperse linear and star polymers, and use these parameter values to predict linear viscoelastic data for a wide range of well-characterized branched polymers [21].

\section{Modifications of the hierarchical model}

We modify the algorithm developed by Larson [13] as explained below, and follow the notation used in that paper. Figure 1 illustrates how the algorithm treats the hierarchical relaxation of a comb-branched polymer, which is composed of arms and backbone segments. At short time after a small step strain, only the arms can relax inward from their tips by early-time fluctuations and late-time retraction. When an arm is fully relaxed, it is conceptually pruned away and replaced by a bead at the branch point, which schematically represents the frictional drag contributed by that arm. Eventually the unrelaxed molecule becomes the equivalent of an " $\mathrm{H}$ " molecule. As more and more arms become completely relaxed, the unrelaxed molecule eventually becomes a "star", and finally a "linear" molecule. The final relaxation then occurs by reptation of an effectively "linear"

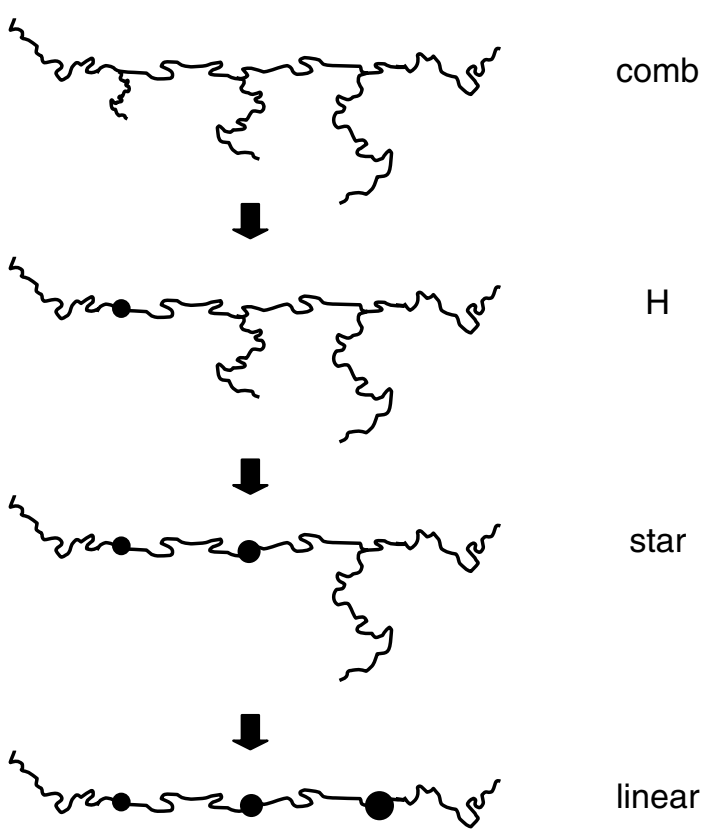

Fig. 1 Conceptualization of algorithm for computing hierarchical relaxation of a comb-branched polymer. See text for details 
chain, which, however, reptates slowly because of the effective beads representing the drag produced by the arms.

In this work, we use the following definitions for the entanglement spacing $M_{\mathrm{e}}$ and the tube diameter $a$ [14]:

$M_{\mathrm{e}}=\frac{4}{5} \frac{\rho R T}{G_{N}^{0}} ; \quad a^{2}=\frac{M_{\mathrm{e}}}{M} N b^{2}$

where $\rho$ is the density of the polymer melt, $R$ is the gas constant, $T$ is the absolute temperature, $G_{N}{ }^{0}$ is the plateau modulus, $M$ is the polymer molecular weight, $N$ is the number of monomers, and $b$ is the monomer-based statistical segment length, defined so that $N b^{2}=\left\langle R^{2}\right\rangle$, where $\left\langle R^{2}\right\rangle$ is the mean-square end-to-end vector of the chain at equilibrium. In the previous paper [13], the definition of $M_{\mathrm{e}}$ was not consistent with the definition of $a$ because of missing the factor of $4 / 5$ in the definition of $M_{\mathrm{e}}$; see [14].

The arm retraction motions are divided into two stages: the first stage consists of the early-time fluctuations, which are limited to small fractional excursions toward the branch point, and the second stage contains the late-time, deep arm retractions, or fluctuations. For the early-time motion, the effective entropic potential impeding the arm retraction is very small. Thus, the motion of the free end can be considered to be a onedimensional Rouse motion. In the previous paper, the early-time fluctuations were not considered. While the "waiting time" prefactor used in the original hierarchical model can include the effect of the early-time fluctuations indirectly through an empirical adjustment of its value, for high molecular weight linear polymers, the resulting prediction for the longest relaxation time is much longer than that obtained experimentally. To avoid this problem, in this work we include directly the early-time fluctuations used by Milner and McLeish [18], which is as follows:

$\tau_{\text {early }}(\xi)=\frac{9}{16} \pi^{3} \tau_{\mathrm{e}} S_{\mathrm{a}}^{4} \xi^{4}$

where $S_{\mathrm{a}} \equiv M_{\mathrm{a}} / M_{\mathrm{e}}$, with $M_{\mathrm{a}}$ the molecular weight of an arm of the star polymer and $\tau_{\mathrm{e}}$ the "equilibration time", i.e., the Rouse time of a single entanglement length of polymer. Here $\xi$ is an arm coordinate that runs from zero to unity as one moves along the contour of the arm from the free end to the branch point. The factor 225/256 in Milner and McLeish's original paper [18] is changed to $9 / 16$ because of our choice of the definition of $M_{\mathrm{e}}$ in Eq. (1). In the original hierarchical model, the relaxation time for the late-time arm retraction was

$\tau_{\text {late }}(\xi)=\tau_{0} S_{\mathrm{a}}^{3 / 2} \exp \left[U_{\mathrm{eff}}(\xi)\right]$

where $\tau_{0}$ is an additional time constant and was determined from fitting the relaxation time of monodisperse star polymers. $U_{\text {eff }}$ is the effective potential for the arm retraction. Thus, the prefactor of the late-time equation in the original hierarchical model was independent of the arm coordinate $\xi$.

The late-time arm retraction time can be obtained from a first-passage time calculation of the diffusion over a barrier (Milner and McLeish [18]):

$\tau_{\text {late }}(\xi)=\frac{L^{2}}{D_{\text {eff }}} \int_{0}^{\xi} d \xi^{\prime} \exp \left[U_{\text {eff }}\left(\xi^{\prime}\right)\right] \int_{-\infty}^{\xi^{\prime}} d \xi^{\prime \prime} \exp \left[-U_{\text {eff }}\left(\xi^{\prime \prime}\right)\right]$

where $L$ is the contour length and $D_{\text {eff }}$ is the effective curvilinear diffusion constant of the retracting arm in the tube. (The lower limit of $-\infty$ on the inner integral allows outward, as well as inward fluctuations of the primitive path). Milner and McLeish have used a more refined prefactor than Eq. (3) for the arm retraction of a star polymer by approximating Eq. (4) as follows [18, 21]:

$$
\begin{aligned}
\tau_{\text {late }}(\xi)=\tau_{\mathrm{e}} S_{\mathrm{a}}^{3 / 2}\left(\frac{\pi^{5}}{6}\right)^{1 / 2} & \frac{\exp \left[U_{\mathrm{eff}}(\xi)\right]}{\left[\xi^{2}(1-\xi)^{2 \alpha}+\left(\frac{1+\alpha}{3 S_{\mathrm{a}}}\right)^{2 \alpha /(\alpha+1)} \Gamma^{-2}\left(\frac{1}{\alpha+1}\right)\right]^{1 / 2}}
\end{aligned}
$$

where $\Gamma$ stands for the gamma function and $\alpha$ is the dilution exponent in the dynamic dilution theory. While we can derive the analytic form of the effective potential and the prefactor in specific systems such as monodisperse stars, star-linear blends, $\mathrm{H}$, and comb polymers $[2,17,18,20]$, it is very difficult to obtain the effective potential analytically for general mixtures of branched polymers. In the original hierarchical model, calculation of the effective potential was not necessary because the time integration only required the differential form of the potential, which could be obtained from the instantaneous concentration of unrelaxed melts, $\Phi(t)$ (see Eq. (10) in the previous paper [13]). This permitted the hierarchical model to deal with general mixtures of branched polymers. However, if the early-time fluctuations are included, we need to calculate the effective potential at every time step in order to construct the crossover equation that splices together the early-time fluctuations and the late-time retraction.

Here we calculate the effective potential for the arm retraction from the general formula:

$U_{\mathrm{eff}}(\xi)=\int_{0}^{\xi} 2 v S_{\mathrm{a}} \Phi\left(\xi^{\prime}\right)^{\alpha} d \xi^{\prime}$ 
where $v=3 / 2$. The value $v=15 / 8$ in Milner and McLeish's original paper [18] and in the previous paper [13] is changed to $3 / 2$ because of our choice of the definition of $M_{\mathrm{e}}$ in Eq. (1). At each time step we calculate the unrelaxed volume fraction $\Phi\left(\xi^{\prime}\right)$, using the arm coordinate $\xi^{\prime}$ as follow:

$\Phi\left(\xi^{\prime}\right)=\sum_{i}^{m}\left[\sum_{j=1}^{n_{\mathrm{a}}(i)} \phi_{\mathrm{a}}(i, j)\left(1-\xi^{\prime}(i, j, t)\right)+\sum_{j=1}^{n_{\mathrm{b}}(i)} \phi_{\mathrm{b}}(i, j)\right]$

where $m, n_{\mathrm{a}}(i)$, and $n_{\mathrm{b}}(i)=n_{\mathrm{a}}(i)-3$ are the number of molecules, the number of arms, and the number of backbone segments, respectively, for molecule $i . \phi_{\mathrm{a}}(i, j)$ and $\phi_{\mathrm{b}}(i, j)$ are the volume fractions of arm and backbone segment for molecule $i$. Note that at any time $t$, the new information needed to compute the value of the potential needed at that time step, namely $\Phi(\xi(t))$, is available from the above equation. The above sums only include arm and backbone segments that have not completely relaxed at time $t$.

There has been much discussion about which value of the "dilution exponent," $\alpha=1$ or $\alpha=4 / 3$, is the more suitable one. In the original version of the hierarchical model [13], $\alpha=1$ was used for the dilution exponent. In this work, however, we use $\alpha=4 / 3$ because we have recently shown that the value of $M_{\mathrm{e}}$ obtained from a bestfit of viscosity of both linear and star polymers is closer to the value determined from the plateau modulus in the case of $\alpha=4 / 3$ [21]. In the modified hierarchical model, the integration in Eq. (6) for the effective potential is performed numerically at every time step, and the late-time arm retraction is then calculated directly from the numerical integration of Eq. (4) with $L^{2} / D_{\text {eff }}=(3 / 2) \pi^{2} S_{\mathrm{a}}{ }^{3} \tau_{\mathrm{e}}$. Thus, the modified hierarchical model does not need the fitting parameter $\tau_{0}$.

In calculating the relaxation spectrum of an arm, we need the crossover equation between the early-time and the late-time functions. To obtain this, we use the equation developed by Milner and McLeish [18], which is

$\tau_{\mathrm{a}}(\xi)=\frac{\tau_{\text {early }}(\xi) \exp \left[U_{\text {eff }}(\xi)\right]}{1+\tau_{\text {early }}(\xi) \exp \left[U_{\text {eff }}(\xi)\right] / \tau_{\text {late }}(\xi)}$

At any time $t$, the arm will have relaxed from its free end to a point $\xi$ obtained by equating $\tau_{\mathrm{a}}$ with $t$; hence

$t=\tau_{\mathrm{a}}(\xi)=\frac{\tau_{\text {early }}(\xi) \exp \left[U_{\text {eff }}(\xi)\right]}{1+\tau_{\text {early }}(\xi) \exp \left[U_{\text {eff }}(\xi)\right] / \tau_{\text {late }}(\xi)}$

The incremental increase in $\xi$ is related to the incremental increase in $\Delta t$ through a differential form of Eq. (8). The new value of $\xi$ is calculated as follows:

$\xi_{\text {new }}=\xi_{\text {old }}+\frac{\Delta t}{d \tau_{\mathrm{a}} / d \xi}$
In this work we use time steps that are equal intervals in time instead of in the logarithm of time, as used in original model, because here we include the early-time fluctuations, which can only be calculated accurately if the time step remains small throughout the integration. (The early time fluctuations affect relaxation even at late time because of the cross-over formula, which is used throughout the calculation).

To account for the sudden decrease of the unrelaxed volume fraction after the reptation time of any linear chains in the mixture, the hierarchical model includes "supertube relaxation" by using a function $\Phi_{\mathrm{ST}}$, which is defined as [20]:

$\Phi_{\mathrm{ST}}=\Phi_{\mathrm{ST}, 0}\left(\frac{t}{t_{0}}\right)^{-1 / 2 \alpha}$

where $t_{0}$ is the time at which supertube relaxation is activated and $\Phi_{\mathrm{ST}, 0}$ is the volume fraction of the unrelaxed material just before the sudden relaxation by reptation occurs. In the original hierarchical model, however, the arm was allowed to relax during the constraint-release Rouse process. The result of this assumption was that the predicted relaxation time was much shorter than that of experimental data in blends of linear and star polymers. In this work, we follow the Milner-McLeish theory [20], where the arm relaxation is not allowed during the constraint-release Rouse process.

The storage and loss moduli $G^{\prime}$ and $G^{\prime \prime}$ are calculated by using

$$
\begin{aligned}
\frac{G^{\prime}(\omega)}{G_{N}^{0}}= & \int_{0}^{1} \frac{\omega^{2} t^{2}}{1+\omega^{2} t^{2}}\left[\Phi_{\mathrm{ST}}(t)\right]^{\alpha} d \Phi(t) \\
& +\alpha \int_{0}^{1} \frac{\omega^{2} t^{2}}{1+\omega^{2} t^{2}} \Phi(t)\left[\Phi_{\mathrm{ST}}(t)\right]^{\alpha-1} d \Phi_{\mathrm{ST}}(t)
\end{aligned}
$$

$$
\begin{aligned}
\frac{G^{\prime \prime}(\omega)}{G_{N}^{0}}= & \int_{0}^{1} \frac{\omega t}{1+\omega^{2} t^{2}}\left[\Phi_{\mathrm{ST}}(t)\right]^{\alpha} d \Phi(t) \\
& +\alpha \int_{0}^{1} \frac{\omega t}{1+\omega^{2} t^{2}} \Phi(t)\left[\Phi_{\mathrm{ST}}(t)\right]^{\alpha-1} d \Phi_{\mathrm{ST}}(t)
\end{aligned}
$$

where $\Phi(t)$ and $\Phi_{\mathrm{ST}}(t)$ are respectively the unrelaxed fraction and the supertube fraction at time $t$ during the relaxation.

All calculations have been carried out on a PC wtth a Pentium III $1 \mathrm{GHz}$ CPU. The computational time required for the new hierarchical model is much greater than that of the old hierarchical model because in the new model the integration of Eq. (6) to obtain the effective potential is carried out at each time step 
and uses a linear time step instead of the logarithmic one used in the old hierarchical model. However, the typical computational time for polydisperse $\mathrm{H}$ - and comb polymers is less than $5 \mathrm{~h}$.

\section{Results and discussion}

Comparison between the hierarchical model and the Milner-McLeish model

In this work we choose 1,4-polybutadienes and 1,4polyisoprenes as model polymers, because for these polymers we can find suitable rheological data in the literature all at the same, or nearly the same, temperature for several different architectures and molecular weights. The parameters needed in the hierarchical model are the plateau modulus $G_{N}{ }^{0}$, the entanglement spacing $M_{\mathrm{e}}$, and the equilibration time $\tau_{\mathrm{e}}$. The value of $G_{N}{ }^{0}$ is obtained from Fetters et al. [5] for polybutadiene and from Pearson et al. [23] for polyisoprene. The value of $M_{\mathrm{e}}$ can be calculated from the value of $G_{N}{ }^{0}$ using Eq. (1). The value of $\tau_{\mathrm{e}}$ is related to the monomeric friction coefficient $\zeta$ and tube diameter $a$ by $\tau_{\mathrm{e}}=\zeta a^{2} M_{\mathrm{e}} /$ $3 \pi^{2} M_{0} k_{\mathrm{B}} T$, where $M_{0}$ is the monomer molecular weight. Therefore, in principle, we can obtain the $\tau_{\mathrm{e}}$ value from the literature values of the tube diameter and the monomeric friction coefficient [3]. We note, however, that the value of $\zeta$ is often based on data from the transition region, and might not be accurate for calculation of slow relaxation processes. In addition, the theory for branched polymers is very sensitive to the values of $M_{\mathrm{e}}$ and $\tau_{\mathrm{e}}$. Thus, in this work, we choose $M_{\mathrm{e}}$ and $\tau_{\mathrm{e}}$ values from the best-fits of zero-shear viscosities of linear and star polymers using the Milner-McLeish theory. For polybutadiene we use $G_{N}{ }^{0}=1.15 \times 10^{5}(\mathrm{~Pa})$, $M_{\mathrm{e}}=1650$ and $\tau_{\mathrm{e}}=3.7 \times 10^{-7}$ (s) at $T=25^{\circ} \mathrm{C}$. Comparison between model predictions using these parameter values and experimental data of polybutadiene is reported in Park and Larson [21].

For polyisoprene we use $G_{N}^{0}=0.44 \times 10^{6} \quad(\mathrm{~Pa})$, $M_{\mathrm{e}}=4054$, and $\tau_{\mathrm{e}}=1.0 \times 10^{-5} \mathrm{~T}=25^{\circ} \mathrm{C}$. Figure 2 shows the zero-shear viscosities of linear and star polyisoprene [Fetters et al. 4, 10]. The lines are the predictions of the Milner-McLeish theory [18, 20]. The Milner-McLeish theory predicts experimental zeroshear viscosity well. While in the case of polybutadiene the value of $M_{\mathrm{e}}=1650$ obtained by best-fitting is slightly higher than the value $\left(M_{\mathrm{e}}=1543\right)$ calculated from the value of $G_{N}{ }^{0}$, for polyisoprene we can use the value $M_{\mathrm{e}}=4054$ obtained using Eq. (1) and the experimental value of $G_{N}{ }^{0}$, to fit the zero-shear viscosities of both linear and star polymers. In this work for each polymer we will use the same parameter values for all hierarchical model predictions of the rheology of all architectures.
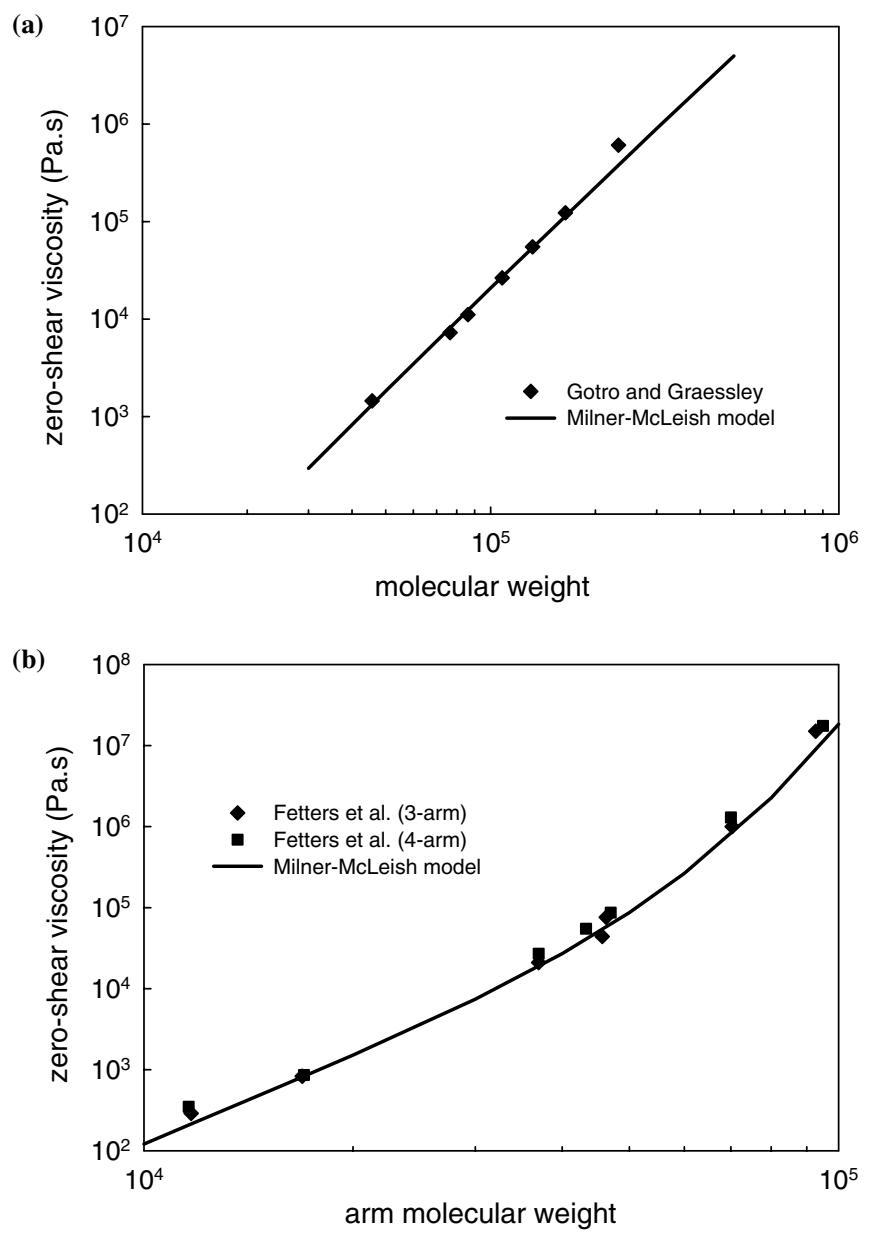

Fig. 2 Zero shear viscosity vs. molecular weight of 1,4-polyisoprenes at $T=25^{\circ} \mathrm{C}$ for a linear and $\mathbf{b}$ star polymers. The symbols are experimental data from Gotro and Graessley [10] and Fetters et al. [4]. The lines are the prediction of the Milner-McLeish theory $[18,19]$

In Fig. 3 we compare the predictions of the MilnerMcLeish theory and of the hierarchical model for the zero-shear viscosities of polybutadiene monodisperse stars using the same parameter values [24, 26, 27, 31]. The dotted line is the prediction of the Milner-McLeish theory and the solid line is the prediction of the hierarchical model. Here and hereafter the term "hierarchical model" refers to the modified version of the theory presented in this paper, not the early version of the theory. As shown in Fig. 3, the prediction of the hierarchical model is almost the same as that of the Milner-McLeish theory and matches well the experimental zero-shear viscosities of monodisperse stars. We note that while the MilnerMcLeish theory uses the approximate form of the firstpassage time equation for the late-time retraction, its predictions are very similar to those of the hierarchical model, in which the first-passage time equation is calculated by numerical integration. 


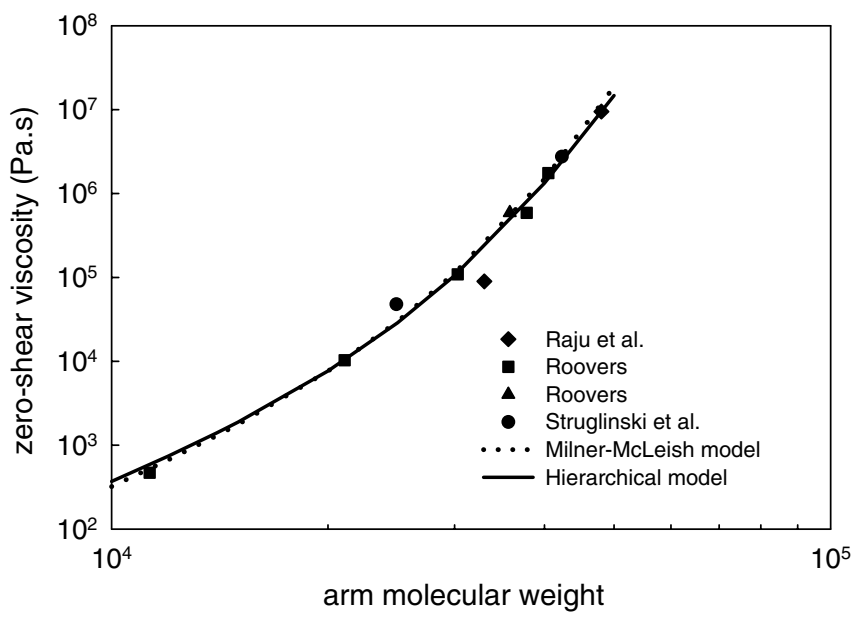

Fig. 3 Comparison between the prediction of the Milner-McLeish theory and that of the hierarchical model for the zero shear viscosity of monodisperse star 1,4-polybutadienes at $T=25^{\circ} \mathrm{C}$ : filled diamond [24]; filled square [26]; $\pi$ [27]; filled circle [31]. The dotted line is the prediction of the Milner-McLeish theory for stars [18] and the solid line is the prediction of the hierarchical model

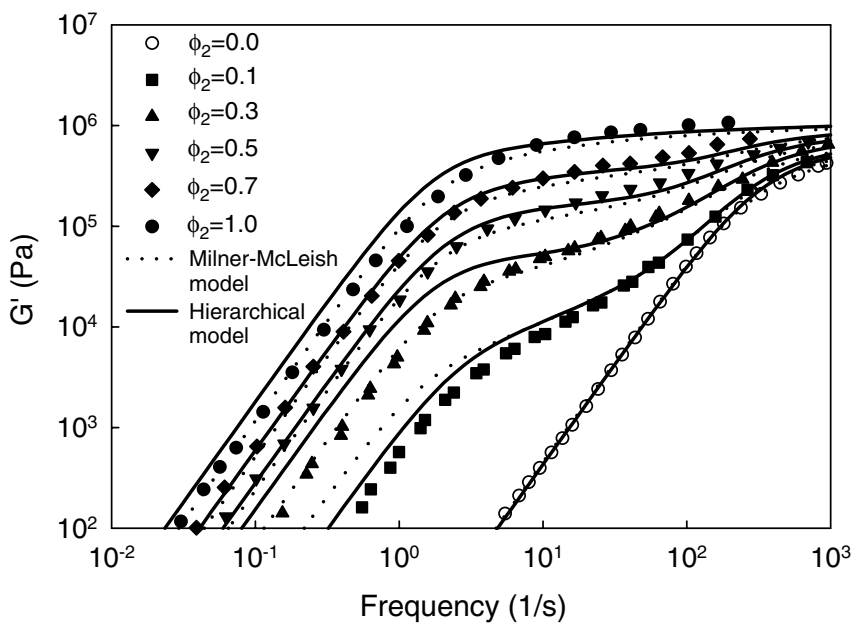

Fig. 4 Storage and loss moduli of bidisperse 1,4-polybutadiene linear melts containing molecular weight 36,800 $\left(M_{1}\right)$ and 168,000 $\left(M_{2}\right)$ mixed at various long-chain volume fractions $\left(\phi_{2}\right)$ at $T=25{ }^{\circ} \mathrm{C}$. The symbols are experimental data from Struglinski and Graessley [30]. The dotted lines are the predictions of the Milner-McLeish theory [22] and the solid lines are the predictions of the hierarchical model

In Fig. 4 we compare the model predictions of the Milner-McLeish theory [19, 22] (dotted lines) and of the hierarchical model (solid lines) with experimental data from Struglinski and Graessley [30] for the storage and loss moduli of linear-linear blends with long-chain volume fractions of $0,0.1,0.3,0.5,0.7$, and 1.0. Since the original Milner-McLeish theory [19] was developed only for monodisperse linear polymers, here we use the extended version of Park and Larson [22] for the bidisperse linear-linear blend. As shown, the model predictions are in good agreement with experimental data. When the early-time fluctuations are included in the modified hierarchical model, the model overpredicts the low-frequency $G^{\prime}$ data for the pure high-molecularweight component, while the Milner-McLeish theory for monodisperse linear polymers predicts this behavior more accurately. The Milner-McLeish theory for linear polymers does not include a cross-over to the late-time retraction, while the hierarchical model includes the latetime retraction even for monodisperse linears, because it was developed to deal with general mixtures of branched polymers, and so the same relaxation mechanisms are applied to all chain ends, whether they are the ends of linear molecules or of branches. If we were to consider only the early-time fluctuations, the hierarchical model predictions could be improved for linear polymers. However, in principle, there appears to be no reason not to include the late-time retraction in the relaxation of linear polymers, even though this inclusion worsens its predictions of data for linear polymers [35].

The experimental data of Struglinski et al. [31], and the corresponding model predictions of the hierarchical model (solid lines) and the Milner-McLeish theory [20, 21] (dotted lines) for the loss modulus are shown in Fig. 5 for star-linear blends with star volume fractions of $0,0.2,0.5,0.75$, and 1.0. As shown, the hierarchicalmodel predictions are very similar to those of the Milner-McLeish theory. While the hierarchical model predictions are not perfect for monodisperse linear polymers, the hierarchical model can capture the relaxation behavior of star-linear blends very well without adjustment of parameter values. We note that in the previous version of the hierarchical model [13], the predictions in the terminal region did not agree well with experimental data because arm retraction was allowed during the constraint-release Rouse motion. The agreement of the predictions of the modified hierarchical model with experimental data for linear and star polymers shows that the modified model can predict the relaxation of linear and star polymers and their blends nearly as well as does the Milner-McLeish model.

\section{Branch point motion}

In the case of $\mathrm{H}$ and comb polymers, the arms are attached to a backbone. The backbone can relax after the arms have completely relaxed. In the relaxation of the backbone, most of the effective friction is concentrated at the branch points. Thus, to obtain more accurate theoretical predictions, the diffusivity of the branch point should be considered, and this is related to the arm retraction time as follows: 


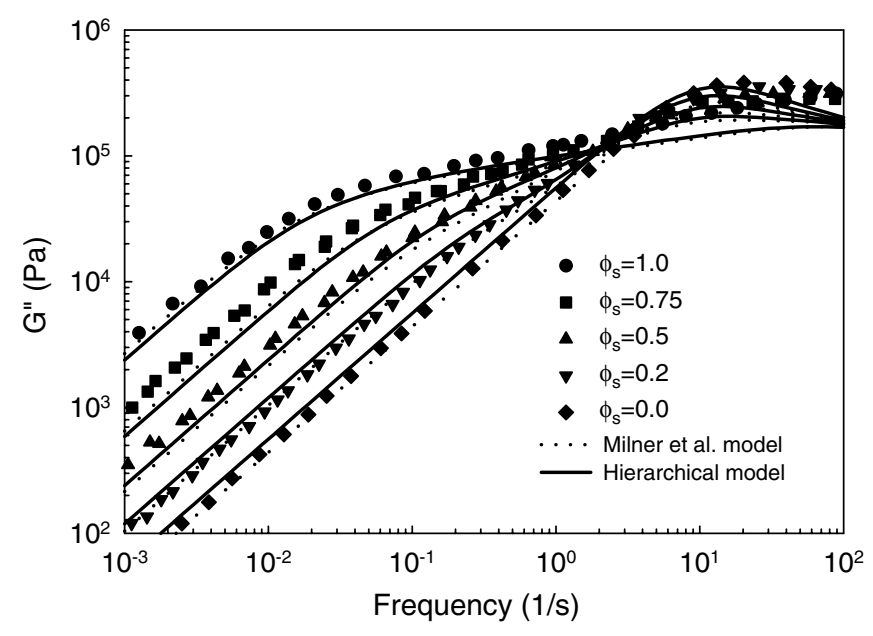

Fig. 5 Loss modulus of bidisperse 1,4-polybutadiene star-linear blends containing linear molecules of molecular weight 100,000 $\left(M_{l}\right)$ and 3-arm stars of total molecular weight $127,000\left(M_{s}\right)$ for various star volume fractions $\left(\phi_{s}\right)$ at $T=25^{\circ} \mathrm{C}$. The symbols are experimental data from Struglinski et al. [31]. The dotted lines are the predictions of the Milner-McLeish theory for star-linear blends [20] and the solid lines are the predictions of the hierarchical model

$D_{\mathrm{br}}=\frac{p^{2} a^{2}}{2 q \tau_{\mathrm{a}}}$

where $p$ is a numerical constant, $a$ is either the undilated or the dilated tube diameter (more on this below), $q$ is the number of dangling arms from each branch point, and $\tau_{\mathrm{a}}$ is the arm retraction time. McLeish et al. [17] compared theoretical predictions with experimental data for polyisoprene $\mathrm{H}$-polymers using a fitted value $p^{2}=1 / 12$ because there is an uncertainty in the mean hopping distance of a branch point. In their calculation, the dilated tube diameter was used in defining the branch point diffusivity. Daniels et al. [2] extended the theory of McLeish et al. [17] to polybutadiene comb polymers. Frischknecht et al. [7] applied the theory of McLeish et al. [17] to polyisoprene asymmetric stars, in which one branch is shorter than the other two equal-length branches. They showed that to obtain agreement with experimental data, the value of $p^{2}$ must be adjusted separately for each asymmetric star. McLeish et al. [17] considered the effect of polydispersity on the terminal relaxation time of an H-polymer and argued that polydispersity increases the terminal relaxation time. However, recently Shanbhag and Larson [28] showed, using a slip link simulation, that the terminal relaxation time of a polydisperse $\mathrm{H}$-polymer is shorter than that of a monodisperse $\mathrm{H}$-polymer of the same weight-average molecular weight.

In this work we test the hierarchical model against data for polyisoprene asymmetric star, $\mathrm{H}$, and polybutadiene comb melts, and examine the effect of polydispersity on their terminal relaxation behavior [2, 7, 17].
Table 1 Molecular characterization of polyisoprene asymmetric star polymers [7]

\begin{tabular}{llll}
\hline Polymer sample & $M_{\mathrm{w}}$ long arm & $M_{\mathrm{w}}$ short arm & $M_{\mathrm{w}} / M_{\mathrm{n}}$ star \\
\hline AS11 & $1.07 \times 10^{5}$ & $1.15 \times 10^{4}$ & 1.02 \\
AS17 & $1.16 \times 10^{5}$ & $1.90 \times 10^{4}$ & 1.01 \\
AS37 & $1.07 \times 10^{5}$ & $3.90 \times 10^{4}$ & 1.01 \\
AS47 & $1.10 \times 10^{5}$ & $4.00 \times 10^{4}$ & 1.02 \\
\hline
\end{tabular}

Table 2 Molecular characterization of polyisoprene H-polymers [17]

\begin{tabular}{lllll}
\hline Polymer sample & $\begin{array}{l}M_{\mathrm{w}} \\
\text { backbone }\end{array}$ & $\begin{array}{l}M_{\mathrm{w}} \\
\text { arm }\end{array}$ & $\begin{array}{l}M_{\mathrm{w}} / M_{\mathrm{n}} \\
\text { backbone }\end{array}$ & $\begin{array}{l}M_{\mathrm{w}} / M_{\mathrm{n}} \\
\text { arm }\end{array}$ \\
\hline H110B20A & $1.11 \times 10^{5}$ & $2.00 \times 10^{4}$ & 1.13 & 1.01 \\
H160B40A & $1.64 \times 10^{5}$ & $4.00 \times 10^{4}$ & 1.30 & 1.05 \\
\hline
\end{tabular}

Table 3 Molecular characterization of polybutadiene comb polymers [2]

\begin{tabular}{llllll}
\hline $\begin{array}{l}\text { Polymer } \\
\text { sample }\end{array}$ & $\begin{array}{l}M_{\mathrm{w}} \\
\text { backbone }\end{array}$ & $\begin{array}{l}M_{\mathrm{w}} \\
\text { arm }\end{array}$ & $\begin{array}{l}M_{\mathrm{w}} / M_{\mathrm{n}} \\
\text { backbone }\end{array}$ & $\begin{array}{l}M_{\mathrm{w}} / M_{\mathrm{n}} \\
\text { arm }\end{array}$ & $\begin{array}{l}\text { Number } \\
\text { of arms }\end{array}$ \\
\hline PBC3 & $1.25 \times 10^{5}$ & $1.77 \times 10^{4}$ & 1.06 & 1.01 & 4.9 \\
PBC5 & $6.39 \times 10^{4}$ & $1.19 \times 10^{4}$ & 1.06 & 1.04 & 8.6 \\
PBC7 & $6.14 \times 10^{4}$ & $2.27 \times 10^{4}$ & 1.03 & 1.07 & 8.4 \\
PBC11 & $6.27 \times 10^{4}$ & $5.80 \times 10^{3}$ & 1.03 & 1.03 & 8.2 \\
\hline
\end{tabular}

The molecular weights of the arms and backbones of these polymers are given in Tables 1,2 and 3 . In the original hierarchical model $p^{2}$ was set to unity, and the undilated tube diameter was used in defining the branchpoint diffusivity. The proper choice of tube diameter to be used in the definition of the branch point diffusivity has been discussed extensively $[2,7,16,17]$. Frischknecht and Milner [6] found that self-diffusion data for hydrogenated polybutadiene star melts are more consistent with diffusive hops distances that scale with the undilated, rather than the dilated tube diameter. In this work we use the undilated tube diameter, and take $p^{2}=1 / 12$ for the calculation of the branch point diffusivity.

\section{Asymmetric star polymers}

The storage and loss moduli of polyisoprene asymmetric stars from Frischknecht et al. [7] are shown in Fig. 6. Here and in what follows, the solid lines are the predictions of the hierarchical model for polydisperse polymers and the dotted lines are those for monodisperse ones, unless otherwise stated. In the hierarchical model, the effect of polydispersity is accounted for by generating an ensemble of molecules to represent the 

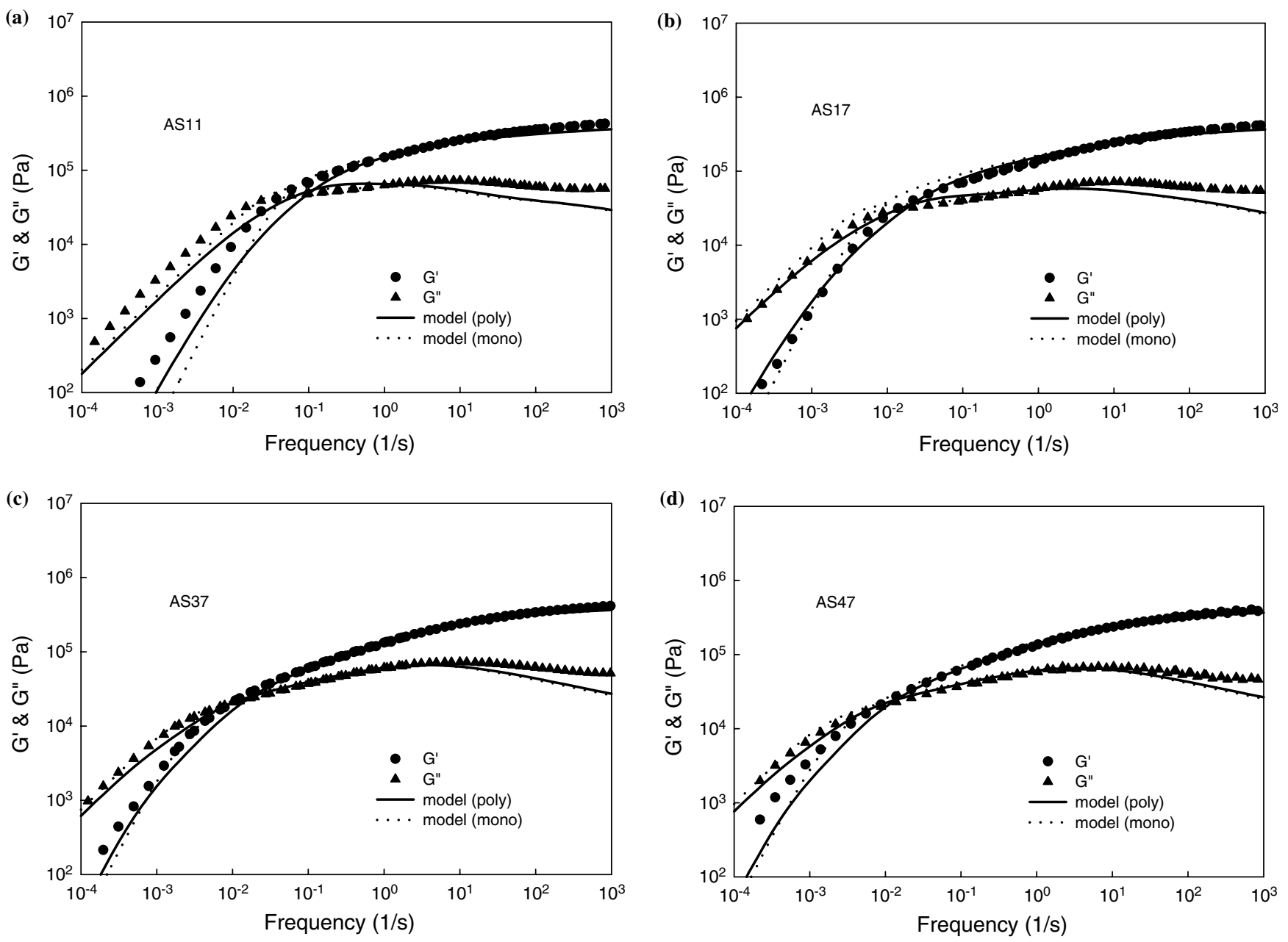

Fig. 6 Storage and loss moduli of polyisoprene asymmetric stars at $T=25^{\circ} \mathrm{C}$. a AS11, b AS17, c AS37, d AS47. The symbols are experimental data from Frischknecht et al. [7]. The solid lines and the dotted lines are the predictions of the hierarchical model with monodisperse and polydisperse arms molecular weights, respectively

molecular weight distribution and then simulating the relaxation of a melt of chains from this ensemble. In this work we have used 1000 molecules for the hierarchical model calculations. Runs with 2000 molecules give nearly the same results. Since for the asymmetric stars only the overall polydispersity was reported, and not the polydispersity of each arm separately, for the prediction with polydispersity, we choose each arm from a lognormal distribution having $M_{\mathrm{w}} / M_{\mathrm{n}}=1.06$ and combine the three arms randomly to make 3-arm asymmetric star polymers with an overall polydispersity of around 1.02, which is close to the reported value. As shown, the predictions with polydispersity are similar to those for the monodisperse predictions. This is consistent with the argument of Frischknecht et al. [7] and the slip-link simulations (Shanbhag and Larson 28] for the asym-

metric star. While Frischknecht et al. [7] found that the value of $p^{2}$ needed to be adjusted to give agreement with experimental data for each asymmetric star, our hierarchical model predictions are in reasonable agreement with experimental data using the same value of $p^{2}$, except for the sample with the shortest arm, AS11. Because the hierarchical model is similar to the theory of Frischknecht et al. [7] the better predictions obtained by the former are due to the different choice of model parameters. Frischknecht et al. [7] chose values of model parameters from fitting data for the symmetric star and linear chain, namely $G_{N}^{0}=0.49 \times 10^{6}(\mathrm{~Pa}), M_{\mathrm{e}}=3641$, and $\tau_{\mathrm{e}}=8.9 \times 10^{-6}$. Because of the factor of $4 / 5$, the value of $M_{\mathrm{e}}=4551$ used by Frischknecht et al. [7] differs from the value $M_{\mathrm{e}}=3641$ in our calculations. (The parameters used by Frischknecht et al. [7] do not predict correctly the experimental data of the monodisperse stars). If we use the same parameter values used by Frischknecht et al. [7] in the hierarchical model predictions, we find, as did Frischknecht et al. [7], that good fits are only obtained if we adjust the value of $p^{2}$ separately for each melt. 


\section{H-polymers}

Figure 7 shows a comparison between hierarchical model predictions and experimental data for polyisoprene $\mathrm{H}$ polymers from McLeish et al. [17] The insets in Fig. 8 contains the predictions of the theory of McLeish et al. [17] for H-polymers, where we used the undilated tube diameter in defining the branch point diffusivity. The parameter values we used for the H-polymer predictions are the same as those for asymmetric stars. The ensemble of polydisperse H-polymers were generated using the same method as employed for the polydisperse asymmetric stars, except that both arm and backbone polydispersities were reported for these polymers and so ensembles of both arms and backbones were generated with log-normal molecular-weight distributions to match
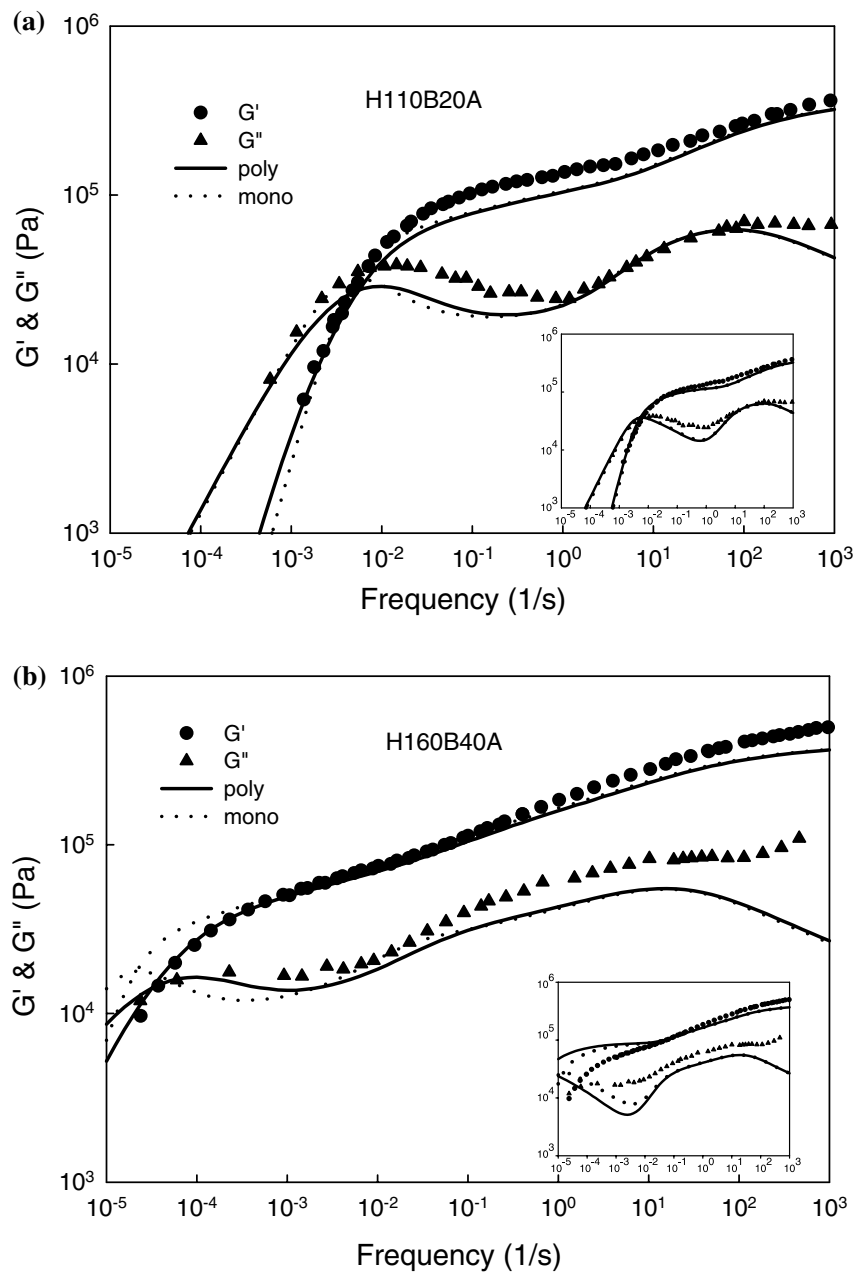

Fig. 7 Storage and loss moduli of polyisoprene H-polymers at $T=25^{\circ} \mathrm{C}$. a H110B20A, b H160B40A. The symbols are experimental data from McLeish et al. [17]. The solid lines and the dotted lines are the predictions of the hierarchical model with monodisperse and polydisperse arm and backbone molecular weights, respectively. The insets are the predictions of the theory of McLeish et al. [17] these polydispersities and then arms from the former ensemble were attached to backbones from the latter ensemble. As shown in Fig. 7, the hierarchical model predicts the experimental data well. These results show that the hierarchical model can predict the relaxation behavior of H-polymers without adjustment of the parameters from the values used for the linear and star polymers.

While in the case of H110B20A, where the arm polydispersity is very small and the predictions with polydispersity are very similar to those without it, for H160B40A, inclusion of polydispersity leads to better agreement with experimental data than does monodispersity. However, as shown in the inset of Fig. 7(b), the polydispersity correction developed by McLeish et al. [17] worsens the predictions, relative to the monodisperse calculation for H160B40A. For an H-polymer, the effect of the backbone polydispersity on the terminal relaxation time is small compared to the effect of the arm polydispersity, but the terminal relaxation time of an $\mathrm{H}$ polymer having polydisperse arms is shorter than that of a monodisperse H-polymer with the same weight average arm and backbone molecular weights as that of the polydisperse $H$. While the decrease of the terminal relaxation time predicted by the hierarchical model for a polydisperse $\mathrm{H}$-polymer is not as drastic as predicted by the slip-link simulations of Shanbhag and Larson [28], the result is qualitatively consistent with their prediction. On the other hand, the polydispersity corrections of McLeish et al. [17] and Frischknecht et al. [7] for $\mathrm{H}$ polymers result in an increase of the terminal relaxation time, the opposite of which is predicted by the hierarchical and the slip-link models. Shanbhag and Larson [28] argued that the diffusivity of the most mobile branch point of H-polymer can be approximated by

$D_{\mathrm{br}}=\frac{p^{2} a^{2}}{2} \sum_{i=1}^{4} \frac{1}{\tau_{\mathrm{a}, i}}$

where $i$ is an index that distinguishes the four arms. Thus, $D_{\text {br }}$ is greatly increased if any one of the four arms is short and is only moderately decreased by the presence of longer arms. Because the perturbation argument used by McLeish et al. [17] and Frischknecht et al. [7] to explain the effect of polydispersity in H-polymer is a mean field approach, it is unable to account properly for the "acceleration" that short branches can induce in the diffusive motion of the backbone. The slip-link model views an entanglement between two chains in a melt as a slip link that permits a sliding motion of both chains, and survives only as long as neither chain has an end that passes through the slip link. Thus, constraint release caused by relaxation of surrounding chains can be captured using creation and destruction of slip-links. The slip-link model offers insight into the drawbacks of the current analytical theory for branched polymers, in which the mobility of the 

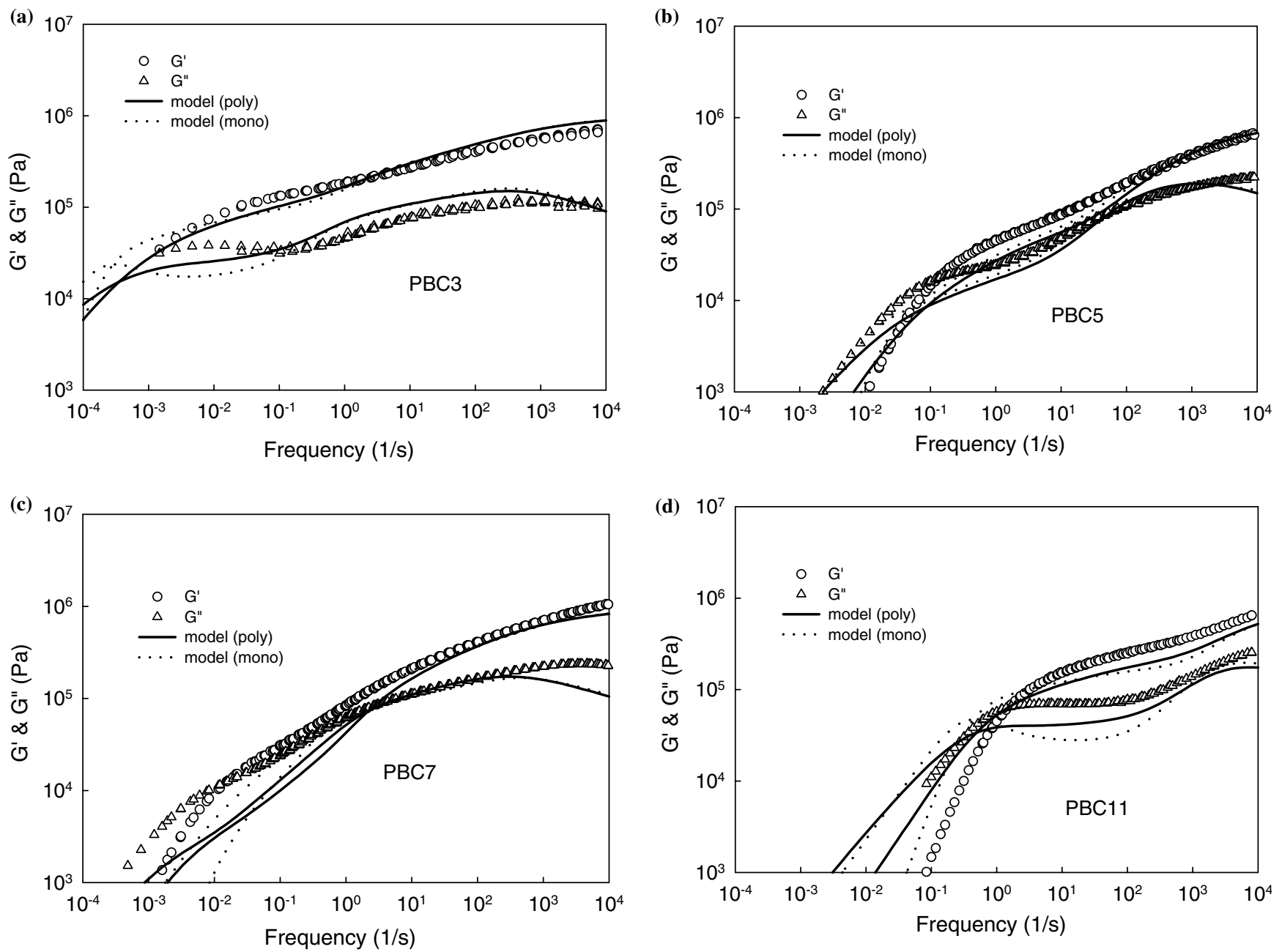

Fig. 8 Storage and loss moduli of polybutadiene combs at $T=25^{\circ} \mathrm{C}$. a $\mathrm{PBC} 3, \mathbf{b} \mathrm{PBC} 5, \mathbf{c} \mathrm{PBC} 7, \mathbf{d} \mathrm{PBC} 11$. The symbols are experimental data from Daniels et al. [2]. The solid lines and the dotted lines are the predictions of the hierarchical model with

branch point is set by the first passage time in a dilating tube. Thus, the slip-link model can in some cases predict the relaxation of branched polymers more accurately than an analytical model such as the hierarchical model. However, the computational time required for simulations with the slip-link model is much larger than that of the hierarchical model. For example, for the comb polymers considered in this paper, the computational time for the slip-link model is longer than 1 month, vs a few hours with the modified hierarchical model.

\section{Comb polymers}

In Fig. 8 we compare the predictions of the hierarchical model with experimental data for polybutadiene combs

from Daniels et al. [2]. Daniels et al. divided the combs into two groups, i.e., combs with a small and large numbers of arms, and used correspondingly different early-time equations and reptation-time expressions for the backbones of the combs. However, the reptation time for the backbone of a comb with a large number of arms (see Eq. (16) in the paper of Daniels et al.) is not correct because it is made proportional to the third power of the number of entanglements in the backbone, rather than of the second power as it should be, because most of drag is contributed by the branch points. Daniels et al. [2] correctly estimated the relaxation time of a comb polymer using $\tau_{\mathrm{a}} L_{\mathrm{b}}{ }^{2} / D_{\mathrm{R}}$, where $L_{\mathrm{b}}$ is the backbone length and $D_{\mathrm{R}}$ is the drag of the reptation. However, for comb polymers, most of drag is contributed by the branch point, so that $D_{\mathrm{R}} \approx p^{2} a^{2} / 2 q \tau_{\text {arm }}$ where $q$ is the number of arms and $\tau_{\text {arm }}$ is the relaxation time of arm. If we neglect the drag contributed by the backbone, we obtain the result $\tau_{\mathrm{d}} \propto q L_{\mathrm{b}}{ }^{2}$ instead of $\tau_{\mathrm{d}} \propto L_{\mathrm{b}}{ }^{3}$, where the latter result would be obtained if friction from the backbone material were more important than the drag due to the arms. 
In the hierarchical model, the expression for the reptation time is the same in both cases of large and small numbers of arms. As shown in Fig. 8, the hierarchical model can predict a priori the qualitative shapes of the curves of $G^{\prime}$ and $G^{\prime \prime}$ for combs. The theory of Daniels et al. [2] cannot predict the relaxation behavior a priori for the comb polymers, but rather the values of the molecular weights and polydispersities of both arms and backbones are adjusted for each polymer to obtain a fit to the data.

The hierarchical model predictions for polydisperse combs are generated using the method explained in the previous paper [13]. Briefly, we allow the arms and the backbone to be polydisperse with a log-normal distribution. The longer backbones contain on average more arms than do shorter ones, i.e., the number of arms follows a Poisson's distribution. For comb polymers, the effect of the polydispersity is complicated because the molecular weights of the backbone segments and of the arms and the positions of branch point are not uniform. Unlike the case of the H-polymer, for comb polymers the hierarchical model predictions do not show quantitative agreement with experimental data even though the polydispersities are included. However, considering that the parameter values obtained from the monodisperse linear and star polymers are not adjusted at all in the predictions for comb polymers and considering the experimental uncertainty in the molecular weight of the arms and backbones and in the number of branches, the predictions of the hierarchical model are qualitatively good.

\section{Conclusions and future directions}

The hierarchical model presented by Larson [13] is modified to give more quantitative agreement of its predictions with experimental data for long-chain branched polymers. For 1,4-polybutadienes and 1,4polyisoprenes, the model predictions are compared with experimental linear viscoelastic data for branched polymers of various structures using a single set of parameter values, which are obtained from fits to experimental data for monodisperse linear and star polymers. The predictions of the hierarchical model are in good agreement with experimental data for linear/ linear and star/linear blends, nearly as good as the predictions of the Milner-McLeish theories. While the model can capture the general trend of the relaxation behavior of asymmetric star, $\mathrm{H}$ - and comb polymers, its predictions do not completely match the experimental data, especially for comb polymers. Nevertheless, considering that a single parameter set has been used for all theoretical predictions for a given polymer chemical type, we can still conclude that the hierarchical model is very promising as a tool for predicting the relaxation of general mixtures of the branched polymers.

The most important issue that remains to be solved in the theoretical modeling of the relaxation of branched polymers is how to deal with the motion of the branch point in a more satisfactory way than has been accomplished so far. Since most of drag for backbone relaxation is contributed by the branch-point motion, an accurate description is essential for quantitative prediction. The existing theories treat branch-point motion as a series of "hops" in either undilated or dilated tubes, but use an empirical prefactor $\left(p^{2}=1 / 12\right)$ which is obtained by data fitting. More microscopic modeling and additional quantitative experimental studies on relaxation of well-defined multiply branched polymers will be required to develop a more satisfying theory.

Acknowledgements $\mathrm{We}$ are grateful to the National Science Foundation, DMR-0096688 and DMR-0072101 for financial support.

\section{References}

1. Crosby BJ, Mangnus M, de Groot W, Daniels R, McLeish TCB (2002) Characterization of long chain branching: dilution rheology of industrial polyethylenes. J Rheol 46:401-426

2. Daniels DR, McLeish TCB, Crosby BJ, Young RN, Fernyhough CM (2001) Molecular rheology of comb polymer melts. 1. Linear viscoelastic response. Macromolecules 34:7025-7033

3. Ferry JD (1980) Viscoelastic properties of polymers. Wiley, New York

4. Fettets LJ, Kiss AD, Pearson DS, Quack GF, Vitus FJ (1993) Rheological behavior of star-shaped polymers. Macromolecules 26:647-654
5. Fetters LJ, Lohse DJ, Richter D, Witten TA, Zirkel A (1994) Connection between polymer molecular weight, density, chain dimensions, and melt viscoelastic properties. Macromolecules 27:4639-4647

6. Frischknecht AL, Milner ST (2000) Self-Diffusion with dynamic dilution in star polymer melts. Macromolecules 33:9764-9768

7. Frischknecht AL, Milner ST, Pryke A, Young RN, Hawkins R, McLeish TCB (2002) Rheology of three-arm asymmetric star polymer melts. Macromolecules $35: 4801-4820$
8. Gabriel C, Münstedt H (2002) Influence of long-chain branches in polyethylenes on linear viscoelastic flow properties in shear. Rheol Acta 41:232-244

9. Gahleitner M (2001) Melt rheology of polyolefins. Prog Polym Sci 26:895-944

10. Gotro JT, Graessley WW (1984) Model hydrocarbon polymers: rheological properties of linear polyisoprenes and hydrogenated polyisoprenes. Macromolecules 17:2767-2775

11. Janzen J, Colby RH (1999) Diagnosing long-chain branching in polyethylenes. J Mol Struct 485-486:569-583 
12. Kratochvil P (2000) Characterization of branched polymers. Macromol Symp 152:279-287

13. Larson RG (2001) Combinatorial rheology of branched polymer melts. Macromolecules 34:4556-4571

14. Larson RG, Sridhar T, Leal LG, McKinley GH, Likhtman AE, McLeish TCB (2003) Definitions of entanglement spacing and time constants in the tube model. J Rheol 47:809-818

15. McLeish TCB (2003) Why, and when, does dynamic tube dilation work for stars? J Rheol 47:177-198

16. McLeish TCB, Larson RG (1998) Molecular constitutive equations for a class of branched polymers: the pompom polymer. J Rheol 42:82-112

17. McLeish TCB, Allgaier J, Bick DK, Bishko G, Biswas P, Blackwell R, Blottiere B, Clarke N, Gibbs B, Groves DJ, Hakiki A, Heenan RK, Johnson JM, Kant R, Read DJ, Young RN (1999) Dynamics of entangled H-polymers: theory, rheology, and neutron-scattering. Macromolecules 32:6734-6758

18. Milner ST, McLeish TCB (1997) Parameter-free theory for stress relaxation in star polymer melts. Macromolecules 30:2159-2166

19. Milner ST, McLeish TCB (1998) Reptation and contour-length fluctuations in melts of linear polymers. Phys Rev Lett 81:725-728
20. Milner ST, McLeish TCB, Young RN, Hakiki A, Johnson JM (1998) Dynamic dilution, constraint-release, and starlinear blends. Macromolecules 31:9345-9353

21. Park SJ, Larson RG (2003) Dilution exponent in the dynamic dilution theory for polymer melts. J Rheol 47:199-211

22. Park SJ, Larson RG (2004) Tube dilation and reptation in binary blends of monodisperse linear polymers. Macromolecules 37:597-604

23. Pearson DS, Mueller SJ, Fetters LJ, Hadjichristidis NJ (1983) Comparison of the rheological properties of linear and star-branched polyisoprenes in shear and elongational flow. Polym Sci Polym Phys Ed 21:2287-2298

24. Raju VR, Menezes EV, Marin G, Graessley WW (1981) Concentration and molecular weight dependence of viscoelastic properties in linear and star polymers. Macromolecules 14:16681676

25. Randall JC (1989) A review of highresolution liquid $\mathrm{C}$-13 nuclear magneticresonance characterizations of ethylene based polymers. J Macromol Sci Rev Macromol Chem Phys C29:201-317

26. Roovers J (1985) Properties of the plateau zone of star-branched polybutadienes and polystyrenes. Polymer 26:1091-1095

27. Roovers J (1987) Tube renewal in the relaxation of 4-arm star polybutadienes in linear polybutadienes. Macromolecules 20:148-152

28. Shanbhag S, Larson RG (2004) A sliplink model of branch-point motion in entangled polymers. Macromolecules (in press)
29. Shroff RN, Mavridis H (2001) Assessment of NMR and rheology for the characterization of LCB in essentially linear polyethylenes. Macromolecules 34:7362-7367

30. Struglinski MJ, Graessley WW (1985) Effects of polydispersity on the linear viscoelastic properties of entangled polymers. 1. Experimental observations for binary mixtures of linear polybutadiene. Macromolecules 18:2630-2643

31. Struglinski MJ, Graessley WW, Fetters LJ (1988) Effects of polydispersity on the linear viscoelastic properties of entangled polymers. 3. Experimental observations on binary mixtures of linear and star polybutadienes. Macromolecules 21:783-789

32. Watanabe $\mathrm{H}$, Matsumiya $\mathrm{Y}$, Inoue $\mathrm{T}$ (2002) Dielectric and viscoelastic relaxation of highly-entangled star polyisoprene; quantitative test of tube dilation model. Macromolecules 35:2339-2357

33. Wood-Adams PM, Dealy JM (2000) Using rheological data to determine the branching level in metallocene polyethylenes. Macromolecules 33:7481-7488

34. Wood-Adams PM, Dealy JM, de Groot AW, Redwine OD (2000) Effect of molecular structure on the linear viscoelastic behavior of polyethylene. Macromolecules 33:7489-7499

35. Ye X, Larson RG, Pattamaprom C, Sridhar T (2003) Extensional properties of monodisperse and bidisperse polystyrene solutions. J Rheol 47:443-468 